\title{
THE ONE-THIRD-TRICK AND SHIFT OPERATORS
}

\author{
RICHARD LECHNER
}

\begin{abstract}
In this paper we obtain a representation as martingale transform operators for the rearrangement and shift operators introduced by T. Figiel in Fig88 and Fig90. The martingale transforms and the underlying sigma algebras are obtained explicitly by combinatorial means. The known norm estimates for those operators are a direct consequence of our representation.
\end{abstract}

\section{Contents}

1. Introduction

2. Preliminaries

3. The One-Third-Trick

4. The Shift Operator $T_{m}$

5. A Martingale Decomposition for $U_{m}$

References

\section{INTRODUCTION}

The proof of the $T(1)$ theorem by T. Figiel proceeds by expanding the integral operator into an absolutely converging series of basic building blocks $T_{m}$ and $U_{m}$, rearranging and shifting the Haar system. This involves the following norm estimates for those building blocks, which T. Figiel obtained by combinatorial means:

$$
\begin{gathered}
\left\|T_{m}: L_{X}^{p} \rightarrow L_{X}^{p}\right\| \leq C\left(\log _{2}(2+|m|)\right)^{\alpha}, \\
\left\|U_{m}: L_{X}^{p} \rightarrow L_{X}^{p}\right\| \leq C\left(\log _{2}(2+|m|)\right)^{\beta},
\end{gathered}
$$

where the constant $C>0$ depends only on $p$, the UMD-constant of $X$ and $0<\alpha, \beta<1$. For the original proof see Fig88 and FW01. See also NS97. and [Mül05]. For extensions to spaces of the homogeneous type see [MP11].

The purpose of the present paper is to obtain a representation of $T_{m}$ and $U_{m}$ as the sum of roughly $\log _{2}(2+|m|)$ martingale transform operators. This is done by combinatorial analysis of the equations defining $T_{m}$ and $U_{m}$.

Our combinatorial analysis exhibits the link of T. Figiel's rearrangement and shift operators to the so called one-third-trick originating in the work of [Wol82], GJ82, [Dav80] and [CWW85].

Date: November 9, 2018.

Partially supported by FWF P20166-N18. 
Related Recent Developments. This article is taken from my Ph.D. thesis [Lec11.

Recently T. P. Hytönen, see Hyt11, presented his own proof of of T. Figiel's vector-valued T(1) theorem, see [Fig90]. The basic aim of T. P. Hytönen in Hyt11 is the same as ours, to find reductions of the general case to certain preferable situations where the so called Figiel compatibility condition is satisfied. To this end the problem in Hyt11 is randomized and the properties of the so called random dyadic partitions of Nazarov, Treil and Volberg, see [NTV97, NTV03] are exploited.

By contrast the proof in the present paper proceeds by finding explicitly those filtrations that turn a given bad combinatorial situation into a good one, such that Figiel's compatibility condition is satisfied. Our reduction is self-contained and develops specific combinatorics of colored dyadic intervals.

Acknowledgment. This article is part of my Ph.D. thesis Lec11 written at the Department of Analysis at the Johannes Kepler University Linz, Austria. I want to thank my Ph.D. advisor P. F. X. Müller for helpful conversations. This paper benefited greatly from extended discussions with Prof. Anna Kamont.

\section{Preliminaries}

The Haar System in $\mathbb{R}$.

Consider the collection of dyadic intervals at scale $j \in \mathbb{Z}$ given by

$$
\mathscr{D}_{j}=\left\{\left[2^{-j} k, 2^{-j}(k+1)[: k \in \mathbb{Z}\},\right.\right.
$$

and the collection of the dyadic intervals

$$
\mathscr{D}=\bigcup_{j \in \mathbb{Z}} \mathscr{D}_{j} .
$$

We define the $L^{\infty}$-normalized Haar system by

$$
h_{[0,1[}(t)=1_{\left[0, \frac{1}{2}[\right.}(t)-1_{\left[\frac{1}{2}, 1[\right.}(t), \quad t \in \mathbb{R},
$$

and for every $I \in \mathscr{D}$ set

$$
h_{I}(t)=h_{[0,1[}\left(\frac{t-\inf I}{|I|}\right), \quad t \in \mathbb{R}
$$

where $1_{A}$ denotes the characteristic function of a set $A$.

Banach Spaces with the UMD-Property.

By $L^{p}(\Omega, \mu ; X)$ we denote the space of functions with values in $X$, Bochnerintegrable with respect to $\mu$. If $\Omega=\mathbb{R}$ and $\mu$ is the Lebesgue measure $|\cdot|$ on $\mathbb{R}$, then set $L_{X}^{p}(\mathbb{R})=L^{p}(\mathbb{R},|\cdot| ; X)$, if unambiguous further abbreviated as $L_{X}^{p}$.

We say $X$ is a UMD space if for every $X$-valued martingale difference sequence $\left\{d_{j}\right\}_{j} \subset L^{p}(\Omega, \mu ; X), 1<p<\infty$, and choice of signs $\varepsilon_{j} \in\{-1,1\}$ one has

$$
\left\|\sum_{j} \varepsilon_{j} d_{j}\right\|_{L^{p}(\Omega, \mu ; X)} \leq \mathscr{U}_{p}(X) \cdot\left\|\sum_{j} d_{j}\right\|_{L^{p}(\Omega, \mu ; X)},
$$

where $\mathscr{U}_{p}(X)$ does not depend on $\varepsilon_{j}$ or $d_{j}$. The constant $\mathscr{U}_{p}(X)$ is called UMDconstant. We refer the reader to Bur81. 


\section{Kahane's Contraction Principle.}

For every Banach space $X, 1 \leq p<\infty$, finite set $\left\{x_{j}\right\}_{j} \subset X$ and bounded sequence of scalars $\left\{c_{j}\right\}_{j}$ we have

$$
\left(\int_{0}^{1}\left\|\sum_{j} r_{j}(t) c_{j} x_{j}\right\|_{X}^{p} \mathrm{~d} t\right)^{1 / p} \leq \sup _{j}\left|c_{j}\right| \cdot\left(\int_{0}^{1}\left\|\sum_{j} r_{j}(t) x_{j}\right\|_{X}^{p} \mathrm{~d} t\right)^{1 / p},
$$

where $\left\{r_{j}\right\}_{j}$ denotes an independent sequence of Rademacher functions. For details see [Kah85. Below we give a short proof, see [Kah85].

Proof. By scaling inequality (2.2), we may assume $\left|c_{j}\right| \leq 1$, for all $j$. We represent each $c_{j}$ as the series $c_{j}=\sum_{k \geq 1} \varepsilon_{j k} 2^{-k}$, with suitable $\varepsilon_{j k} \in\{ \pm 1\}$ and observe

$$
\begin{aligned}
\left(\int_{0}^{1}\left\|\sum_{j} r_{j}(t) c_{j} x_{j}\right\|_{X}^{p} \mathrm{~d} t\right)^{1 / p} & \leq \sum_{k \geq 1} 2^{-k}\left(\int_{0}^{1}\left\|\sum_{j} r_{j}(t) \varepsilon_{j k} x_{j}\right\|_{X}^{p} \mathrm{~d} t\right)^{1 / p} \\
& =\left(\int_{0}^{1}\left\|\sum_{j} r_{j}(t) x_{j}\right\|_{X}^{p} \mathrm{~d} t\right)^{1 / p} .
\end{aligned}
$$

The last equality holds true since $\sum_{j} r_{j}(t) \varepsilon_{j k} x_{j}$ has the same distribution as $\sum_{j} r_{j}(t) x_{j}$ for all choices of signs $\varepsilon_{j k}$.

\section{The Martingale Inequality of Stein - Bourgain's Version.}

Let $(\Omega, \mathcal{F}, \mu)$ be a probability space, and let $\mathcal{F}_{1} \subset \ldots \subset \mathcal{F}_{m} \subset \mathcal{F}$ denote an increasing sequence of $\sigma$-algebras. For every choice of $f_{1}, \ldots, f_{m} \in L^{p}(\Omega, \mu ; X)$, $1<p<\infty$, let $r_{1}, \ldots, r_{m}$ denote independent Rademacher functions, then

$$
\int_{0}^{1}\left\|\sum_{i=1}^{m} r_{i}(t) \mathbb{E}\left(f_{i} \mid \mathcal{F}_{i}\right)\right\|_{L^{p}(\Omega, \mu ; X)} \mathrm{d} t \leq C \cdot \int_{0}^{1}\left\|\sum_{i=1}^{m} r_{i}(t) f_{i}\right\|_{L^{p}(\Omega, \mu ; X)} \mathrm{d} t,
$$

where $C$ depends only on $p$ and $\mathscr{U}_{p}(X)$. The scalar valued version of (2.3) by E. M. Stein can be found in Ste70]. The vector valued extension is due to J. Bourgain [Bou86]. A proof may be found in [FW01].

\section{Additional Notation.}

Let $\mathscr{N}$ be a collection of nested sets, then $\pi_{\mathscr{N}}: \mathscr{N} \rightarrow \mathscr{N}$ is defined as follows. Let $K \in \mathscr{N}$ and then $\pi_{\mathscr{N}}(K)$ is the smallest element with respect to inclusion of the collection $\{M \in \mathscr{N}: K \subsetneq M\}$. In most cases we will omit the subscript and explicitly state to which nested collection we refer.

Given a collection of Lebesgue measurable sets $\mathscr{L}$, the collection of Lebesgue measurable sets $\sigma$-algebra $(\mathscr{L})$ denotes the smallest sigma algebra containing $\mathscr{L}$.

\section{The ONE-Third-TRICK}

We will introduce and investigate two variants of one-third-shift operators, that is the bilateral alternating one-third-shift operator and the unilateral one-third-shift operator. First we will introduce the bilateral alternating one-third-shift operator $S$ given by $S\left(h_{I}\right)=h_{\sigma(I)}$, see (3.4). Roughly speaking, $\sigma$ shifts intervals, say for example having length 1 , to the right by one third of their length, so in our instance by $\frac{1}{3}$. The intervals having length $\frac{1}{2}$ are then shifted by one third of their size to the left, so by $\frac{1}{6}$. Hence the relative translation of two successive levels of dyadic intervals amounts to a total of $\frac{1}{2}$, thus yielding a nested collection of intervals, again. This is illustrated in Figure 1 on the following page In Theorem 3.2 we establish that $S: L_{X}^{p} \longrightarrow L_{X}^{p}$ is an isomorphism by means of Bourgain's version of Stein's martingale inequality. 


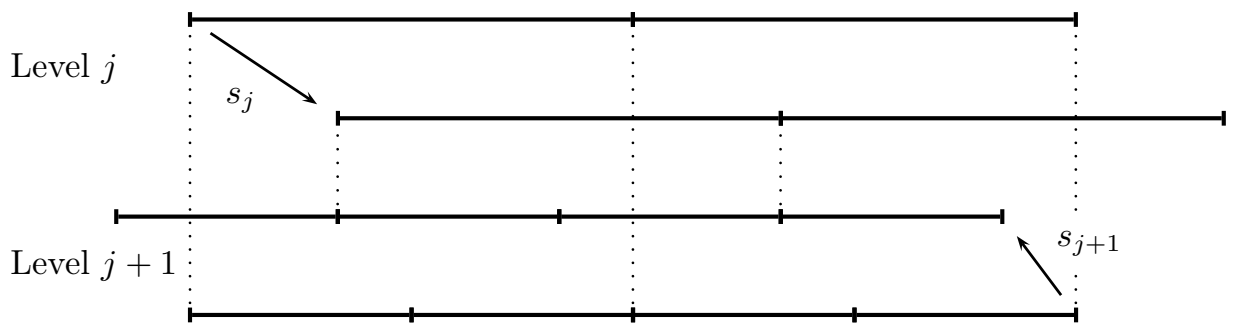

FIGURE 1. One-third-shift of two consecutive levels of intervals. In this illustration $j$ is even.

Finally, we will consider the unilateral variants $S_{0}$ and $S_{1}$ of the one-third-shift operator, and establish in Theorem 3.3 that both are isomorphic maps from $L_{X}^{p}$ to itself, as well.

The one-third-trick originates with the work of [Wol82], GJ82] and [CWW85].

\subsection{Bilateral Alternating One-Third-Shift.}

For every $j \in \mathbb{Z}$ let

$$
s_{j}=(-1)^{j} 2^{-j} / 3
$$

and define

$$
s(I)=s_{j},
$$

for all intervals $I$ having measure $|I|=2^{-j}$. Then define the one-third-shift map

$$
\sigma(I)=I+s(I)
$$

and the one-third-shift operator

$$
S\left(h_{I}\right)=h_{\sigma(I)},
$$

where by $h_{\sigma(I)}$ we denote the function $h_{\sigma(I)}(x)=h_{I}(x-s(I))$. The one-third-shift of dyadic intervals for two consecutive levels is illustrated in Figure 1.

From this picture one can see that the collection of one-third-shifted dyadic intervals $\sigma(\mathscr{D})$ is nested, and $\mathscr{D} \cap \sigma(\mathscr{D})=\emptyset$. Note that if a one-third-shifted dyadic interval $J \in \sigma(\mathscr{D})$ is contained in a non-shifted interval $I \in \mathscr{D}$, then $\operatorname{dist}\left(J, I^{c}\right) \geq$ $|J| / 3$. For every given interval $I \in \mathscr{D}$ exists a unique one-third-shifted interval $J \in \sigma(\mathscr{D}),|J|=|I| / 2$ being contained in $I$. First observe that for every $j \in \mathbb{Z}$ and $I \in \mathscr{D}_{j}$ we have

$$
\begin{gathered}
\#\left\{J \in \sigma\left(\mathscr{D}_{j+1}\right): J \cap I \neq \emptyset\right\}=3, \\
\#\left\{J \in \sigma\left(\mathscr{D}_{j+1}\right): J \subset I\right\}=1 .
\end{gathered}
$$

So we can define $\omega(I)$ by

$$
\omega(I)=J, \quad \text { where } J \in \sigma(\mathscr{D}),|J|=|I| / 2 \text { and } J \subset I,
$$

see Figure 2 on the facing page

Note the basic properties summarized in

Lemma 3.1. The following statements are true.

(i) $\sigma(\mathscr{D})$ is a nested collection of dyadic intervals, and $\mathscr{D} \cap \sigma(\mathscr{D})=\emptyset$.

(ii) $\omega: \mathscr{D} \longrightarrow \sigma(\mathscr{D})$ is well defined and injective.

(iii) Let $I \in \mathscr{D}$, then $\omega(I) \subset I$.

(iv) For every $I \in \mathscr{D}$ we have $\operatorname{dist}\left(\omega(I), I^{c}\right)=|I| / 6$. 


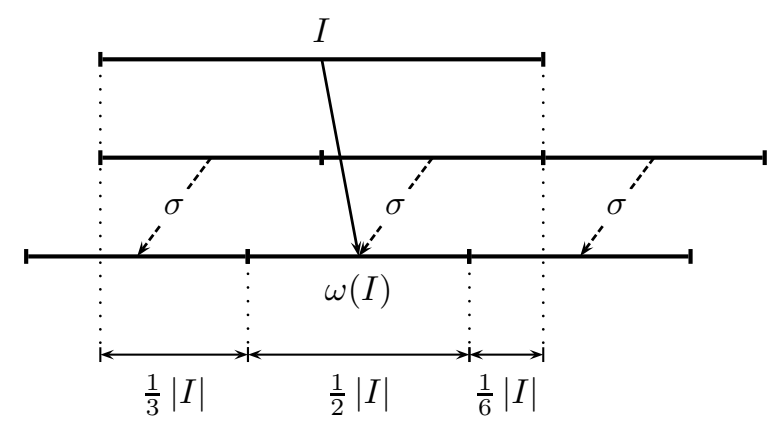

Figure 2. The interval $I$ has measure $|I|=2^{-j}$ with $j$ being even.

(v) Let $I, J \in \mathscr{D},|I|=|J|$, then $\operatorname{dist}(\omega(I), \omega(J))<|\omega(I)|$ if and only if $I=J$. (vi) For all $I \in \mathscr{D}$ we have the identity $\sigma(I)=\omega(I) \cup(\omega(I)+\operatorname{sign}(s(I)) \cdot|\omega(I)|)$.

Proof. The assertions are easily verified.

We need to build up some more notation. For all $j \in \mathbb{Z}$ and

$$
u=\sum_{I \in \mathscr{D}} u_{I} h_{I}|I|^{-1}
$$

let $(u)_{j}$ restrict the function $u$ to level $j$, precisely

$$
(u)_{j}=\sum_{I \in \mathscr{D}_{j}} u_{I} h_{I}|I|^{-1} .
$$

If we define

$$
\mathbb{I}(u)_{j}=\sum_{I \in \mathscr{D}_{j}} u_{I} 1_{I}|I|^{-1},
$$

then we find due to Kahane's contraction principle (2.2) that

$$
\int_{0}^{1}\left\|\sum_{j \in \mathbb{Z}} r_{j}(t)(u)_{j}\right\|_{L_{X}^{p}} \mathrm{~d} t=\int_{0}^{1}\left\|\sum_{j \in \mathbb{Z}} r_{j}(t) \mathbb{I}(u)_{j}\right\|_{L_{X}^{p}} \mathrm{~d} t .
$$

The following theorem establishes that the one-third-shift operator $S: L_{X}^{p} \longrightarrow$ $L_{X}^{p}$ is an isomorphism.

Theorem 3.2. Let $1<p<\infty$ and $X$ a Banach space with the UMD-property, then there exists a constant $C>0$ such that

$$
\frac{1}{C}\|u\|_{L_{X}^{p}} \leq\|S u\|_{L_{X}^{p}} \leq C\|u\|_{L_{X}^{p}},
$$

for all $u \in L_{X}^{p}$. The constant $C$ depends only on $\mathscr{U}_{p}(X)$.

Proof. Let $u=\sum_{I \in \mathscr{D}} u_{I} h_{I}|I|^{-1} \in L_{X}^{p}$ be fixed throughout this proof and set

$$
v=\sum_{I \in \mathscr{D}} u_{I} h_{\omega(I)}|\omega(I)|^{-1}
$$


Note that $\{\omega(I): I \in \mathscr{D}\}$ is nested, see Lemma 3.1 assertion (ii) and (iii). Observe we have due to Lemma 3.1] assertion (iii) that $\mathbb{I}(u)_{j}=\mathbb{E}\left(\mathbb{I}(v)_{j} \mid \mathscr{D}_{j}\right)$, so the UMDproperty and Kahane's contraction principle (2.2) yield

$$
\begin{aligned}
\|u\|_{L_{X}^{p}} & \lesssim \int_{0}^{1}\left\|\sum_{j \in \mathbb{Z}} r_{j}(t)(u)_{j}\right\|_{L_{X}^{p}} \mathrm{~d} t \\
& =\int_{0}^{1}\left\|\sum_{j \in \mathbb{Z}} r_{j}(t) \mathbb{I}(u)_{j}\right\|_{L_{X}^{p}} \mathrm{~d} t \\
& =\int_{0}^{1}\left\|\sum_{j \in \mathbb{Z}} r_{j}(t) \mathbb{E}\left(\mathbb{I}(v)_{j} \mid \mathscr{D}_{j}\right)\right\|_{L_{X}^{p}} \mathrm{~d} t .
\end{aligned}
$$

Now we apply Stein's martingale inequality (2.3) followed by identity (3.8) to pass from $\mathbb{I}(v)_{j}$ to $(v)_{j}$, so

$$
\begin{aligned}
\int_{0}^{1}\left\|\sum_{j \in \mathbb{Z}} r_{j}(t) \mathbb{E}\left(\mathbb{I}(v)_{j} \mid \mathscr{D}_{j}\right)\right\|_{L_{X}^{p}} \mathrm{~d} t & \lesssim \int_{0}^{1}\left\|\sum_{j \in \mathbb{Z}} r_{j}(t) \mathbb{I}(v)_{j}\right\|_{L_{X}^{p}} \mathrm{~d} t \\
& =\int_{0}^{1}\left\|\sum_{j \in \mathbb{Z}} r_{j}(t)(v)_{j}\right\|_{L_{X}^{p}} \mathrm{~d} t .
\end{aligned}
$$

Recalling definition (3.4) and applying Kahane's contraction principle in consideration of $\omega(I) \subset \sigma(I)$ (see identity (vi) in Lemma 3.1), we estimate

$$
\int_{0}^{1}\left\|\sum_{j \in \mathbb{Z}} r_{j}(t)(v)_{j}\right\|_{L_{X}^{p}} \mathrm{~d} t \leq 2 \cdot \int_{0}^{1}\left\|\sum_{j \in \mathbb{Z}} r_{j}(t)(S u)_{j}\right\|_{L_{X}^{p}} \mathrm{~d} t,
$$

and the UMD-property implies

$$
\int_{0}^{1}\left\|\sum_{j \in \mathbb{Z}} r_{j}(t)(v)_{j}\right\|_{L_{X}^{p}} \mathrm{~d} t \lesssim\|S u\|_{L_{X}^{p}} .
$$

Thus, collecting the inequalities yields

$$
\|u\|_{L_{X}^{p}} \lesssim\|S u\|_{L_{X}^{p}}
$$

One can repeat the preceding argument with the roles of $u$ and $S u$ interchanged and obtain the converse inequality

$$
\|S u\|_{L_{X}^{p}} \lesssim\|u\|_{L_{X}^{p}}
$$

\subsection{Unilateral One-Third-Shift.}

We now introduce modified versions $\sigma_{0}$ and $\sigma_{1}$ of the one-third-shift map $\sigma$. To this end we define $\sigma_{0}, \sigma_{1}: \mathscr{D} \longrightarrow \sigma(\mathscr{D})$,

$$
\begin{array}{ll}
\sigma_{0}(I)=J, & \text { where } J \in \sigma(\mathscr{D}),|J|=|I| \text { and } \sup J \in I, \\
\sigma_{1}(I)=J, & \text { where } J \in \sigma(\mathscr{D}),|J|=|I| \text { and inf } J \in I,
\end{array}
$$

see Figure 3 on the next page. This induces the one-third-shift operators $S_{0}$ and $S_{1}$ given by the linear extension of

$$
\begin{aligned}
& S_{0}\left(h_{I}\right)=h_{\sigma_{0}(I)}, \quad I \in \mathscr{D}, \\
& S_{1}\left(h_{I}\right)=h_{\sigma_{1}(I)}, \quad I \in \mathscr{D} \text {. }
\end{aligned}
$$

Observe that we have either

$$
\sigma(I)=\sigma_{0}(I) \quad \text { or } \quad \sigma(I)=\sigma_{1}(I),
$$




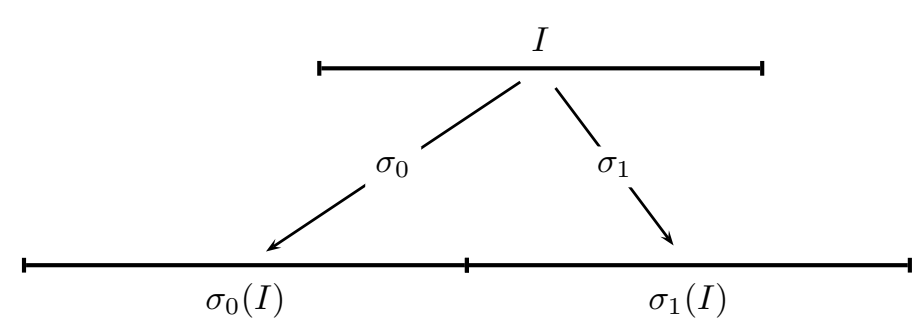

Figure 3. Unilateral one-third-shifts $\sigma_{0}$ and $\sigma_{1}$ applied to $I \in \mathscr{D}$. In this picture the one-third-shift map $\sigma$ shifts to the right, so $\sigma_{1}(I)=\sigma(I)$.

depending on the direction in which $\sigma$ one-third-shifts the interval $I$. Anyhow, we can see that

$$
\left|I \cap \sigma_{0}(I)\right| \geq \frac{1}{3}|I|, \quad\left|I \cap \sigma_{1}(I)\right| \geq \frac{1}{3}|I|,
$$

for all $I \in \mathscr{D}$. The proof of Theorem 3.2 on page 5 with minor modifications yields Theorem 3.3 below.

Theorem 3.3. Let $1<p<\infty$ and $X$ a Banach space with the UMD-property, then there exists a constant $C>0$ such that

$$
\begin{aligned}
& \frac{1}{C}\|u\|_{L_{X}^{p}} \leq\left\|S_{0} u\right\|_{L_{X}^{p}} \leq C\|u\|_{L_{X}^{p}}, \\
& \frac{1}{C}\|u\|_{L_{X}^{p}} \leq\left\|S_{1} u\right\|_{L_{X}^{p}} \leq C\|u\|_{L_{X}^{p}},
\end{aligned}
$$

for all $u \in L_{X}^{p}$. The constant $C$ depends only on $\mathscr{U}_{p}(X)$.

Proof. Define $\omega_{0}$ and $\omega_{1}$ by

$$
\begin{array}{ll}
\omega_{0}(I)=J, & \text { where } J \in \sigma(\mathscr{D}),|J|=|I| / 4 \text { and } \sup J=\sup \sigma_{0}(I), \\
\omega_{1}(I)=J, & \text { where } J \in \sigma(\mathscr{D}),|J|=|I| / 4 \text { and } \inf J=\inf \sigma_{1}(I),
\end{array}
$$

for all $I \in \mathscr{D}$. Now all we need to do is repeat the proof of Theorem 3.3 with $\omega$ replaced by $\omega_{\delta}$ in order to estimate $S_{\delta}$, for each $\delta \in\{0,1\}$.

\section{The Shift Operator $T_{m}$}

Here we define $16+4 \cdot \log _{2}(|m|), m \neq 0$ collections of the Haar system, so that on each such subcollection $T_{m}$ acts as a martingale transform operator on either the dyadic grid or the one-third-shifted dyadic grid. In section 3 we established that changing the dyadic grid to the one-third-shifted dyadic grid is an isomorphism. Thus we may assume that $T_{m}$ is representable as a martingale transform operator on each of the $16+4 \cdot \log _{2}(|m|)$ subcollections, which yields the well known estimate

$$
\left\|T_{m}: L_{X}^{p} \rightarrow L_{X}^{p}\right\| \leq C \cdot\left(\log _{2}(2+|m|)\right)^{\alpha},
$$

for some $0<\alpha<1$, established by T. Figiel in Fig88.

Define the shift map $\tau_{m}, m \in \mathbb{Z}$ by

$$
\tau_{m}(I)=I+m|I|,
$$

for all $I \in \mathscr{D} \cup \sigma(\mathscr{D})$. This induces the shift operator $T_{m}$, given by

$$
T_{m} h_{I}=h_{\tau_{m}(I)},
$$


for all $I \in \mathscr{D} \cup \sigma(\mathscr{D})$. It is crucial that the one-third-shift operator $S$ defined in (3.4) and the shift operator $T_{m}$ commute, that is the identity

$$
\left(S \circ T_{m}\right)(u)=\left(T_{m} \circ S\right)(u),
$$

for all $u \in L_{X}^{p}$. Analogously, we have that

$$
\begin{aligned}
& \left(S_{0} \circ T_{m}\right)(u)=\left(T_{m} \circ S_{0}\right)(u), \\
& \left(S_{1} \circ T_{m}\right)(u)=\left(T_{m} \circ S_{1}\right)(u),
\end{aligned}
$$

for all $u \in L_{X}^{p}$, see (3.9), (3.10), (3.11) and (3.12).

We aim at splitting the dyadic intervals $\mathscr{D}$ into collections $\mathscr{B}_{i}^{(\delta)}$, such that we may bound $T_{m} \circ S^{\delta}$ on functions supported on $\sigma^{\delta}\left(\mathscr{B}_{i}^{(\delta)}\right), \delta \in\{0,1\}$. Note that if $\delta=0$, then $S^{\delta}=\mathrm{Id}$ and $\sigma^{\delta}=\mathrm{Id}$.

Given a shift width $m \in \mathbb{Z}, m \neq 0$, we will partition the dyadic intervals $\mathscr{D}$ into $16+4 \cdot \log _{2}(|m|)$ disjoint collections denoted by $\mathscr{B}_{i}^{(\delta)}$. The collections are constructed in such way that for each $i$ and $\delta \in\{0,1\}$ fixed, we have that whenever $I \in \mathscr{B}_{i}^{(\delta)}$, the intervals $\sigma^{\delta}(I)$ and $\left(\tau_{m} \circ \sigma^{\delta}\right)(I)$ share the same dyadic predecessor with respect to the collection $\sigma^{\delta}\left(\mathscr{B}_{i}^{(\delta)}\right)$. The details are elaborated in Lemma 4.1 below.

Lemma 4.1. For every integer $m \in \mathbb{Z}, m \neq 0$ let $\tau_{m}$ denote the map given by

$$
\tau_{m}(I)=I+m|I|,
$$

for all $I \in \mathscr{D} \cup \sigma(\mathscr{D})$, see (4.1).

Then there exist a constant $K(m) \leq 7+2 \cdot \log _{2}(|m|)$ and disjoint collections of dyadic intervals $\mathscr{B}_{i}^{(\delta)}, 0 \leq i \leq K(m), \delta \in\{0,1\}$ with

$$
\mathscr{D}=\bigcup_{\delta \in\{0,1\}} \bigcup_{i=0}^{K(m)} \mathscr{B}_{i}^{(\delta)},
$$

such that

$$
\left\{I, \tau_{m}(I), I \cup \tau_{m}(I): I \in \sigma^{\delta}\left(\mathscr{B}_{i}^{(\delta)}\right)\right\}
$$

is a nested collection of sets, for all $0 \leq i \leq K(m)$ and $\delta \in\{0,1\}$.

Proof. Due to symmetry we may assume that $m \geq 1$, and we set $K(m)=K(-m)$, if $m \leq-1$. So fix a shift width $m \geq 2$ and a $\lambda \geq 4$ such that

$$
2^{\lambda-3} \leq m<2^{\lambda-2}
$$

and define $L(m)=\lambda-1$. If $m=1$, then let $\lambda=4$ and set $L(1)=3$. Now we split $\mathscr{D}$ into disjoint collections $\mathscr{A}_{i}, 0 \leq i \leq L(m)$, by omitting $L(m)$ consecutive levels of $\mathscr{D}$. More precisely, for every $0 \leq i \leq L(m)$ we define

$$
\mathscr{A}_{i}=\bigcup_{j \in \mathbb{Z}}\left\{I \in \mathscr{D}:|I|=2^{-(\lambda \cdot j+i)}\right\} .
$$

Next we want to divide each of the $\mathscr{A}_{i}$ into two collections $\mathscr{A}_{i}^{(0)}$ and $\mathscr{A}_{i}^{(1)}$, such that every $I \in \mathscr{A}_{i}^{(0)}$ has the same predecessor in $\mathscr{A}_{i}^{(0)}$ as $\tau_{m}(I)$, and $\mathscr{A}_{i}^{(0)}$ is maximal. As a consequence, the collection $\mathscr{A}_{i}^{(1)}$ consists all intervals $I$ such that $I$ and $\tau_{m}(I)$ do not share the same predecessor. But, if we apply the one-third-shift map $\sigma$ to the collection $\mathscr{A}_{i}^{(1)}$, then every $I \in \sigma\left(\mathscr{A}_{i}^{(1)}\right)$ has the same predecessor in 


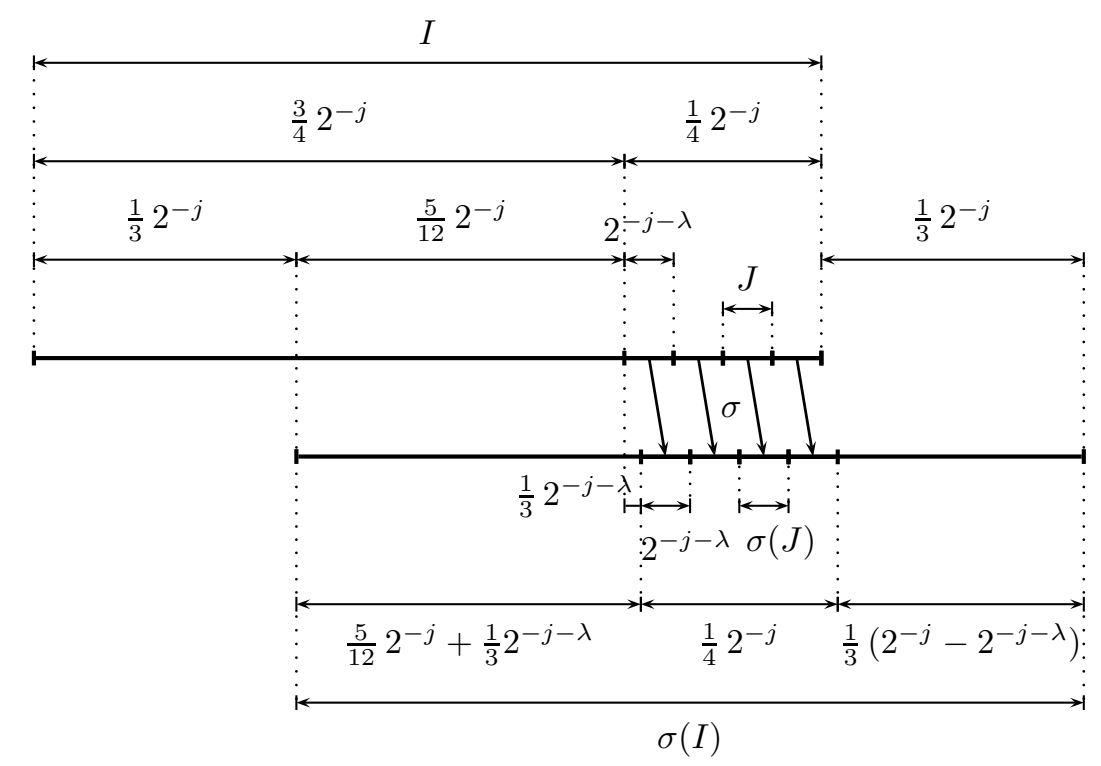

FiguRE 4. The one-third-shift map $\sigma$ acting on $I \in \mathscr{D},|I|=2^{-j}$ and $J \in \mathscr{D},|J|=2^{-j-\lambda}$, where $J \subset I$ and $\tau_{m}(J) \cap I=\emptyset$. In this picture $\lambda$ is even.

$\sigma\left(\mathscr{A}_{i}^{(1)}\right)$ as $\tau_{m}(I)$. We will now construct these two collections. To this end let $\mathscr{G}$ denote one of the collections $\mathscr{A}_{i}, \sigma\left(\mathscr{A}_{i}\right), 0 \leq i \leq L(m)$ and define

$$
\begin{aligned}
& \mathscr{C}_{0}(\mathscr{G}, I)=\left\{J \in \mathscr{G}:|J|=2^{-\lambda}|I|, J \subset I \text { and } \tau_{m}(J) \subset I\right\}, \\
& \mathscr{C}_{1}(\mathscr{G}, I)=\left\{J \in \mathscr{G}:|J|=2^{-\lambda}|I|, J \subset I \text { and } \tau_{m}(J) \cap I=\emptyset\right\} .
\end{aligned}
$$

Revisiting the definition of the one-third-shift map (3.3) and considering the restriction (4.7) one can see that

$$
\sigma\left(\mathscr{C}_{1}\left(\mathscr{A}_{i}, I\right)\right) \subset \mathscr{C}_{0}\left(\sigma\left(\mathscr{A}_{i}\right), \sigma(I)\right)
$$

for all $I \in \mathscr{A}_{i}, 0 \leq i \leq L(m)$. This means that all intervals $J \in \sigma\left(\mathscr{C}_{1}\left(\mathscr{A}_{i}, I\right)\right)$ are such that $J$ and $\tau_{m}(J)$ share $\sigma(I)$ as common predecessor with respect to the collection $\sigma\left(\mathscr{A}_{i}^{(1)}\right)$. In Figure 4 one can see the action of the one-third-shift map $\sigma$ on the collection $\mathscr{A}_{i}$. Now define for every $0 \leq i \leq L(m)$ the following collections of dyadic intervals

$$
\begin{aligned}
& \mathscr{A}_{i}^{(0)}=\bigcup\left\{\mathscr{C}_{0}\left(\mathscr{A}_{i}, I\right): I \in \mathscr{A}_{i}\right\}, \\
& \mathscr{A}_{i}^{(1)}=\mathscr{A}_{i} \backslash \mathscr{A}_{i}^{(0)} .
\end{aligned}
$$

Finally, for all $0 \leq i \leq L(m)$ and $\delta \in\{0,1\}$ we split $\mathscr{A}_{i}^{(\delta)}$ into two disjoint collections

such that

$$
\mathscr{B}_{i}^{(\delta)} \quad \text { and } \quad \mathscr{B}_{i+L(m)+1}^{(\delta)},
$$

$$
\mathscr{B}_{i}^{(\delta)} \cap \tau_{m}\left(\mathscr{B}_{i}^{(\delta)}\right)=\emptyset
$$

for all $0 \leq i \leq K(m)$ and $\delta \in\{0,1\}$, where we set $K(m)=2 \cdot L(m)+1$. Considering (4.7) and $L(m)=\lambda-1$ we find that $K(m) \leq 7+2 \cdot \log _{2}(m)$. For this purpose consider the collection

$$
\mathscr{E}=\left\{\tau_{k}(I): I \in \mathscr{D}, \inf I=0,0 \leq k \leq m-1\right\},
$$


and observe that

$$
\mathscr{D}=\bigcup_{\substack{j \in \mathbb{Z} \\ j \text { even }}} \tau_{j \cdot m}(\mathscr{E}) \cup \bigcup_{\substack{j \in \mathbb{Z} \\ j \text { odd }}} \tau_{j \cdot m}(\mathscr{E})=\mathscr{D}_{\text {even }} \cup \mathscr{D}_{\text {odd }} .
$$

Now define the collections

$$
\begin{aligned}
\mathscr{B}_{i}^{(\delta)} & =\mathscr{A}_{i}^{(\delta)} \cap \mathscr{D}_{\text {even }}, \\
\mathscr{B}_{i+L(m)+1}^{(\delta)} & =\mathscr{A}_{i}^{(\delta)} \cap \mathscr{D}_{\text {odd }},
\end{aligned}
$$

for all $0 \leq i \leq L(m)$ and $\delta \in\{0,1\}$.

With regard to (4.10), (4.9) and noting that $\tau_{m}(I) \in \mathscr{D}_{\text {odd }}$ if and only if $I \in$

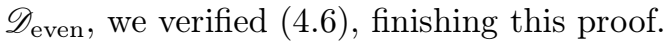

Remark 4.2. Note that we actually proved the slightly stronger result

$$
I \cup \tau_{m}(I) \subset \pi^{\lambda}(I)
$$

for all $I \in \sigma^{\delta}\left(\mathscr{B}_{i}^{(\delta)}\right), 0 \leq i \leq K(m), \delta \in\{0,1\}$. Conceive the predecessor map $\pi$ with respect to $\sigma^{\delta}(\mathscr{D})$. To be more precise let $I \in \sigma^{\delta}(\mathscr{D})$. Then $\pi(I)$ is the unique interval $J \in \sigma^{\delta}(\mathscr{D})$ such that $J \supset I$, and $\pi^{\lambda}=\pi \circ \cdots \circ \pi$.

As the combinatorial Lemma 4.1 on page 8 exhibits the link between the shift map $\tau_{m}$, the one-third-shift map $\sigma$ and Figiel's compatibility condition (4.6), the subsequent Theorem 4.3 will translate the combinatorial results into analytical results, exhibiting the link between the shift operator $T_{m}$, the one-third-shift operator $S$ and martingale transform operators.

In the following context understand that $1<p<\infty, X$ is a Banach space with the UMD-property and $m \in \mathbb{Z}, m \neq 0$. Now we define the projections $P_{i}^{(\delta)}: L_{X}^{p} \longrightarrow L_{X}^{p}$, associated with the collections $\mathscr{B}_{i}^{(\delta)}$ in Lemma 4.1 on page 8

$$
P_{i}^{(\delta)} u=\sum_{I \in \mathscr{B}_{i}^{(\delta)}}\left\langle u, h_{I}\right\rangle h_{I}|I|^{-1},
$$

for all $0 \leq i \leq K(m), \delta \in\{0,1\}$ and $u \in L_{X}^{p}$. The Banach space $X$ having the UMD-property implies uniform bounds on the projections $P_{i}^{(\delta)}$. Note the identity

$$
u=\sum_{\delta \in\{0,1\}} \sum_{i=0}^{K(m)} P_{i}^{(\delta)} u
$$

holds true for all $u \in L_{X}^{p}$, since the collections $\mathscr{B}_{i}^{(\delta)}, 0 \leq i \leq K(m), \delta \in\{0,1\}$ form a partition of $\mathscr{D}$, see Lemma 4.1 .

Exploiting that the one-third-shift operator $S$ is an isomorphism on $L_{X}^{p}$ (see Theorem 3.2 , we will now estimate the shift operator $T_{m}$ on the range of each $P_{i}^{(\delta)}$ in the subsequent theorem.

Theorem 4.3. Let $1<p<\infty$ and $X$ be a Banach space with the UMD-property. Then for every $m \in \mathbb{Z}, 0 \leq i \leq K(m)$ and $\delta \in\{0,1\}$ the inequality

$$
\left\|T_{m} \circ P_{i}^{(\delta)} u\right\|_{L_{X}^{p}} \leq C \cdot\left\|P_{i}^{(\delta)} u\right\|_{L_{X}^{p}},
$$

holds true for all $u \in L_{X}^{p}$, where the constant $C$ depends only on $\mathscr{U}_{p}(X)$. The projections $P_{i}^{(\delta)}, 0 \leq i \leq K(m), \delta \in\{0,1\}$ are defined according to (4.16), and $K(m) \leq 7+2 \cdot \log _{2}(1+|m|)$. 
Proof. Note that due to symmetry once we established (4.18) for $m \geq 1$, the theorem is proved.

Recalling the properties of the partition $\mathscr{B}_{i}^{(\delta)}, 0 \leq i \leq K(m), \delta \in\{0,1\}$ of $\mathscr{D}$, see Lemma 4.1 on page 8 , and we know that the collection

$$
\left\{I, \tau_{m}(I), I \cup \tau_{m}(I): I \in \sigma^{\delta}\left(\mathscr{B}_{i}^{(\delta)}\right)\right\}
$$

is nested, for all $0 \leq i \leq K(m)$ and $\delta \in\{0,1\}$. Throughout this proof let $m \in \mathbb{Z}$, $0 \leq i \leq K(m), \delta \in\{0,1\}$ and $u \in P_{i}^{(\delta)}\left(L_{X}^{p}\right)$ be fixed. According to (4.16) we may assume that $u$ has the representation

$$
u=\sum_{I \in \mathscr{B}_{i}^{(\delta)}} u_{I} h_{I}|I|^{-1}
$$

For every $J \in \sigma^{\delta}(\mathscr{D})$ let

$$
A^{(\delta)}(J)=J \cup \tau_{m}(J),
$$

and for all $j \in \mathbb{Z}$ define the collection

$$
\mathscr{A}_{j}^{(\delta)}=\left\{A^{(\delta)}(J): J \in \sigma^{\delta}\left(\mathscr{D}_{j}\right)\right\} .
$$

Then specify the filtration $\left\{\mathscr{F}_{j}^{(\delta)}\right\}_{j}$ by

$$
\mathscr{F}_{j}^{(\delta)}=\sigma \text {-algebra }\left(\bigcup_{i \leq j} \mathscr{A}_{i}^{(\delta)}\right),
$$

and observe that due to (4.19) every $A^{(\delta)}(J), J \in \sigma^{\delta}\left(\mathscr{D}_{j}\right)$ is an atom for $\mathscr{F}_{j}^{(\delta)}$. The one-third-shift operator is given by

$$
S^{\delta} u=\sum_{I \in \mathscr{B}_{i}^{(\delta)}} u_{I} h_{\sigma^{\delta}(I)}|I|^{-1}=\sum_{J \in \sigma^{\delta}\left(\mathscr{B}_{i}^{(\delta)}\right)} u_{\sigma^{-\delta}(J)} h_{J}|J|^{-1},
$$

see (3.4) for details. We recall the notation

$$
(u)_{j}=\sum_{|I|=2^{-j}} u_{I} h_{I}|I|^{-1} \text { and } \mathbb{I}(u)_{j}=\sum_{|I|=2^{-j}} u_{I} 1_{I}|I|^{-1},
$$

and note that

$$
\left\|T_{m} S^{\delta} u\right\|_{L_{X}^{p}} \approx \int_{0}^{1}\left\|\sum_{j \in \mathbb{Z}} r_{j}(t) \mathbb{I}\left(T_{m} S^{\delta} u\right)_{j}\right\|_{L_{X}^{p}} \mathrm{~d} t
$$

see (3.6), (3.7) and (3.8). Obviously, $\mathbb{I}\left(T_{m} S^{\delta} u\right)_{j} \leq 2 \cdot \mathbb{E}\left(\mathbb{I}\left(S^{\delta} u\right)_{j} \mid \mathscr{F}_{j}^{(\delta)}\right)$, hence Kahane's contraction principle and Bourgain's version of Stein's martingale inequality yield

$$
\begin{aligned}
\int_{0}^{1}\left\|\sum_{j \in \mathbb{Z}} r_{j}(t) \mathbb{I}\left(T_{m} S^{\delta} u\right)_{j}\right\|_{L_{X}^{p}} \mathrm{~d} t & \leq \int_{0}^{1}\left\|\sum_{j \in \mathbb{Z}} r_{j}(t) 2 \cdot \mathbb{E}\left(\mathbb{I}\left(S^{\delta} u\right)_{j} \mid \mathscr{F}_{j}^{(\delta)}\right)\right\|_{L_{X}^{p}} \mathrm{~d} t \\
& \lesssim \int_{0}^{1}\left\|\sum_{j \in \mathbb{Z}} r_{j}(t) \mathbb{I}\left(S^{\delta} u\right)_{j}\right\|_{L_{X}^{p}} \mathrm{~d} t \\
& \approx\left\|S^{\delta} u\right\|_{L_{X}^{p}} .
\end{aligned}
$$

Combining the latter two estimates with Theorem 3.2 on page 5 proves

$$
\left\|T_{m} S^{\delta} u\right\|_{L_{X}^{p}} \lesssim\|u\|_{L_{X}^{p}}
$$

According to (4.3) the shift operator $T_{m}$ and the one-third-shift operator $S$ commute, so we have the identity

$$
T_{m} u=\left(S^{-\delta} \circ T_{m} \circ S^{\delta}\right)(u),
$$


and we obtain by an application of Theorem 3.2 on page 5

$$
\left\|T_{m} u\right\|_{L_{X}^{p}} \lesssim\left\|\left(T_{m} \circ S^{\delta}\right)(u)\right\|_{L_{X}^{p}} .
$$

We conclude the proof by joining (4.25) and (4.24).

Remark 4.4. By slightly adjusting the construction of $\mathscr{B}_{i}^{(\delta)}$ we could replace Bourgain's version of Stein's martingale inequality by the martingale transforms in Fig88. Proposition 2, Step 0] in order to obtain (4.24). To this end we will basically have to replace $\lambda$ by $\lambda+1$ and redefine $\mathscr{C}_{0}$ and $\mathscr{C}_{1}$ as follows

$$
\begin{aligned}
\mathscr{C}_{0}\left(I, \mathscr{A}_{i}\right)= & \left\{J \in \mathscr{A}_{i}:|J|=2^{-\lambda}|I|, J \subset I_{0} \text { and } \tau_{m}(J) \subset I_{0}\right\} \\
& \cup\left\{J \in \mathscr{A}_{i}:|J|=2^{-\lambda}|I|, J \subset I_{1} \text { and } \tau_{m}(J) \subset I_{1}\right\}, \\
\mathscr{C}_{1}\left(I, \mathscr{A}_{i}\right)= & \left\{J \in \mathscr{A}_{i}:|J|=2^{-\lambda}|I|, J \subset I_{0} \text { and } \tau_{m}(J) \cap I_{0}=\emptyset\right\} \\
& \cup\left\{J \in \mathscr{A}_{i}:|J|=2^{-\lambda}|I|, J \subset I_{1} \text { and } \tau_{m}(J) \cap I_{1}=\emptyset\right\},
\end{aligned}
$$

confer (4.8) and (4.9). This results in the collection

$$
\left\{J_{0}, \tau_{m}(J)_{0}, J_{1}, \tau_{m}(J)_{1}, J \cup \tau_{m}(J): J \in \sigma^{\delta}\left(\mathscr{B}_{i}^{(\delta)}\right)\right\}
$$

being nested for all $0 \leq i \leq K(m)$ and $\delta \in\{0,1\}$. With this modifications let us define

$$
d_{J, 1}^{(\delta)}=\frac{1}{2}\left(h_{J}+h_{\tau_{m}(J)}\right) \quad \text { and } \quad d_{J, 2}^{(\delta)}=\frac{1}{2}\left(h_{J}-h_{\tau_{m}(J)}\right),
$$

for all $J \in \sigma^{\delta}\left(\mathscr{B}_{i}^{(\delta)}\right)$. Since (4.26) is nested, $\left\{d_{J, 1}^{(\delta)}, d_{J, 2}^{(\delta)}: J \in \sigma\left(\mathscr{B}_{i}^{(\delta)}\right)\right\}$ forms a martingale difference sequence. Observe $h_{J}=d_{J, 1}^{(\delta)}+d_{J, 2}^{(\delta)}$ and $h_{\tau_{m}(J)}=d_{J, 1}^{(\delta)}-d_{J, 2}^{(\delta)}$, hence we may swap $h_{J}$ and $h_{\tau_{m}(J)}$ without using Bourgain's version of Stein's martingale inequality.

\section{A Martingale Decomposition for $U_{m}$}

In this section we will decompose the Haar system into $24+6 \cdot \log _{2}(|m|)$ subcollections, so that on each fixed subcollection the rearrangement operator $U_{m}$ is either a martingale transform operator itself or the sum of two martingale transform operators. To be more precise, the total amount of subcollections on which we will estimate parts of $U_{m}$ that act as martingale transform operators will be $40+10 \cdot \log _{2}(|m|)$. This gives immediately the estimate Fig88

$$
\left\|U_{m}: L_{X}^{p} \rightarrow L_{X}^{p}\right\| \leq C \cdot\left(\log _{2}(2+|m|)\right)^{\beta},
$$

for some $0<\beta<1$.

The operator $T_{m}$ is easier to analyze than $U_{m}$. This is mainly due to the observation that $\left\{T_{m} h_{I}\right\}_{I \in \mathscr{A}}$ is a martingale difference sequence for any choice of $\mathscr{A} \subset \mathscr{D}$, whereas whether $\left\{U_{m} h_{I}\right\}_{I \in \mathscr{B}}$ forms a martingale difference sequence strongly depends on the choice of $\mathscr{B} \subset \mathscr{D}$. Making use of the one-third-shift operators introduced in Section 3, we will decompose the operator $U_{m}$ into the five parts

$$
U_{m}=U_{m} \circ P^{(0)}+\sum_{\varepsilon \in\{0,1\}}\left(A_{m}^{(\varepsilon)}+B_{m}^{(\varepsilon)}\right) \circ P^{(1, \varepsilon)}
$$

each of which behaves like $T_{m}$. Some parts of this decomposition will be well localized, whereas others are widespread, see Figures [5, 6] and 7 .

In (4.1) we defined the shift map $\tau_{m}$ for every $m \in \mathbb{Z}$ by

$$
\tau_{m}(I)=I+m|I|,
$$


for all $I \in \mathscr{D} \cup \sigma(\mathscr{D})$. Now we introduce the shift operator $U_{m}$ by setting

$$
U_{m} h_{I}=1_{\tau_{m}(I)}-1_{I},
$$

for all $I \in \mathscr{D} \cup \sigma(\mathscr{D})$. Essentially the same method we used to bound $T_{m}$ for functions supported on the collections $\mathscr{B}_{i}^{(0)}, 0 \leq i \leq K(m)$ qualifies for estimating $U_{m}$. This is primarily due to the fact that $\left\{U_{m} h_{I}: I \in \mathscr{B}_{i}^{(0)}\right\}$ forms a martingale difference sequence, which is ensured by Lemma 4.1. The main obstacle is to estimate $U_{m}$ on $\mathscr{B}_{i}^{(1)}$, since $\left\{U_{m} h_{I}: I \in \mathscr{B}_{i}^{(1)}\right\}$ is not a martingale difference sequence. The remedy to this problem is the martingale difference sequence decomposition of $U_{m}$ into

where

$$
U_{m} h_{I}=a_{I}^{(\varepsilon)}+b_{I}^{(\varepsilon)}-b_{\tau_{m}(I)}^{(\varepsilon)}, \quad I \in \mathscr{B}_{i}^{(1, \varepsilon)}
$$

$$
\begin{aligned}
& \mathscr{B}_{i}^{(1,0)}=\left\{I \in \mathscr{B}_{i}^{(1)}: \inf \tau_{m}(I) \neq \inf \pi^{\lambda}\left(\tau_{m}(I)\right)\right\}, \\
& \mathscr{B}_{i}^{(1,1)}=\left\{I \in \mathscr{B}_{i}^{(1)}: \inf \tau_{m}(I)=\inf \pi^{\lambda}\left(\tau_{m}(I)\right)\right\} .
\end{aligned}
$$

Recall that given $\delta \in\{0,1\}$ and an interval $I \in \sigma^{\delta}(\mathscr{D})$, the interval $\pi(I)$ is the unique $J \in \sigma^{\delta}(\mathscr{D})$ such that $J \supset I$, and $\pi^{\lambda}=\pi \circ \cdots \circ \pi$. The collections $\left\{a_{I}^{(\varepsilon)}: I \in\right.$ $\left.\mathscr{B}_{i}^{(1, \varepsilon)}\right\}$ and $\left\{b_{I}^{(\varepsilon)}, b_{\tau_{m}(I)}^{(\varepsilon)}: I \in \mathscr{B}_{i}^{(1, \varepsilon)}\right\}$ are martingale difference sequences, each, see Theorem 5.1. This is what enables us to treat $U_{m}$ like $T_{m}$, which is elaborated in Theorem 5.4 .

First, we define $\alpha_{0}, \alpha_{1}: \mathscr{D} \longrightarrow \sigma(\mathscr{D})$,

$$
\begin{aligned}
& \alpha_{0}(I)=\sigma_{0}(I), \\
& \alpha_{1}(I)=\sigma_{1}(I),
\end{aligned}
$$

where $\sigma_{0}$, and $\sigma_{1}$ are given by (3.9) and (3.10) in Subsection 3.2. Secondly, define the maps $\beta_{0}, \beta_{1}$ and $\beta$ by

$$
\begin{aligned}
\beta_{0}(I) & =\alpha_{0}(I) \backslash I, \\
\beta_{1}(I) & =\alpha_{1}(I) \cap I, \\
\beta(I) & =\beta_{0}(I) \cup \beta_{1}(I) .
\end{aligned}
$$

Finally, $\gamma_{0}, \gamma_{1}$ and $\gamma$ are given by

$$
\begin{aligned}
\gamma_{0}(I) & =\tau_{-1}(I), \\
\gamma_{1}(I) & =I, \\
\gamma(I) & =\gamma_{0}(I) \cup \gamma_{1}(I) .
\end{aligned}
$$

The functions $\alpha_{0}, \alpha_{1}, \beta_{0}$ and $\beta_{1}$ are visualized in Figure 5 on the following page.

With $m \in \mathbb{Z}, m \geq 1$ fixed, we introduce the functions

$$
\begin{array}{ll}
a_{I}^{(0)}=1_{\alpha_{0}\left(\tau_{m}(I)\right)}-1_{\alpha_{0}(I)}, & I \in \mathscr{D}, \\
b_{I}^{(0)}=1_{\beta_{0}(I)}-1_{\beta_{1}(I)}, & I \in \mathscr{D},
\end{array}
$$

and

$$
\begin{array}{ll}
a_{I}^{(1)}=1_{\alpha_{1}\left(\tau_{m}(I)\right)}-1_{\alpha_{1}(I)}, & I \in \mathscr{D}, \\
b_{I}^{(1)}=1_{I \backslash \beta_{1}(I)}-1_{I \backslash \beta_{0}(I)}, & I \in \mathscr{D} .
\end{array}
$$

see Figures [6] and 7 We define the operators $A_{m}^{(\varepsilon)}, B^{(\varepsilon)}$ and $B_{m}^{(\varepsilon)}$ as the linear 

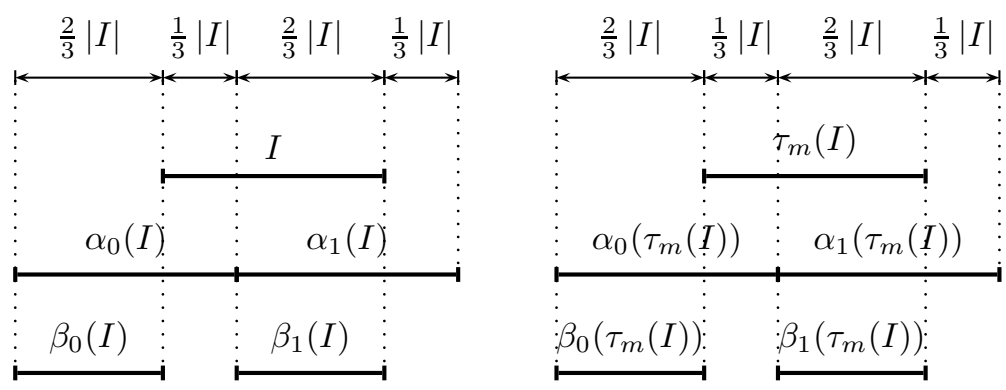

Figure 5. The support functions $\alpha_{0}, \alpha_{1}, \beta_{0}, \beta_{1}$ for $I$ and $\tau_{m}(I)$.

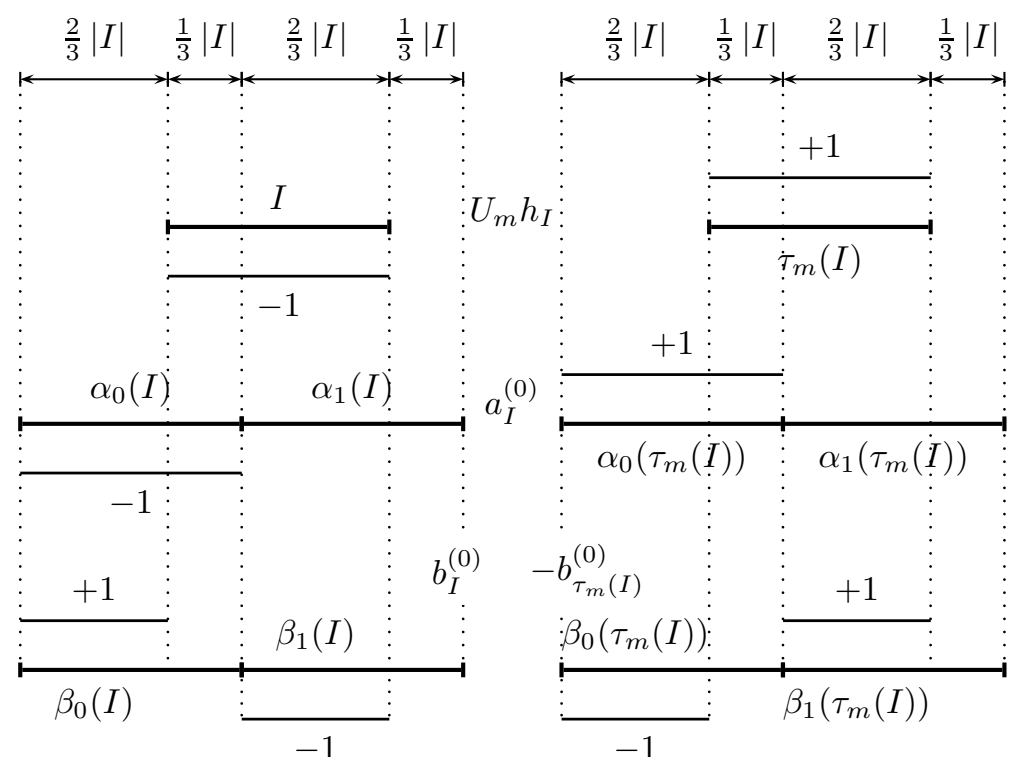

Figure 6. Martingale decomposition of $U_{m}$ to the left.

extension of

$$
\begin{array}{ll}
A_{m}^{(\varepsilon)} h_{I}=a_{I}^{(\varepsilon)}, & I \in \mathscr{D}, \\
B^{(\varepsilon)} h_{I}=b_{I}^{(\varepsilon)}, & I \in \mathscr{D}, \\
B_{m}^{(\varepsilon)} h_{I}=b_{I}^{(\varepsilon)}-b_{\tau_{m}(I)}^{(\varepsilon)}, & I \in \mathscr{D},
\end{array}
$$

for $\varepsilon \in\{0,1\}$. Note the identities

$$
U_{m}=A_{m}^{(\varepsilon)}+B_{m}^{(\varepsilon)}=A_{m}^{(\varepsilon)}+B^{(\varepsilon)}-B^{(\varepsilon)} \circ T_{m},
$$

hold true for $\varepsilon \in\{0,1\}$, see (5.10), (5.11), (5.12), (5.13) and Figures 6 and 7 .

Now we split the collections $\mathscr{B}_{i}^{(1)}$ into

$$
\mathscr{B}_{i}^{(1)}=\mathscr{B}_{i}^{(1,0)} \cup \mathscr{B}_{i}^{(1,1)},
$$




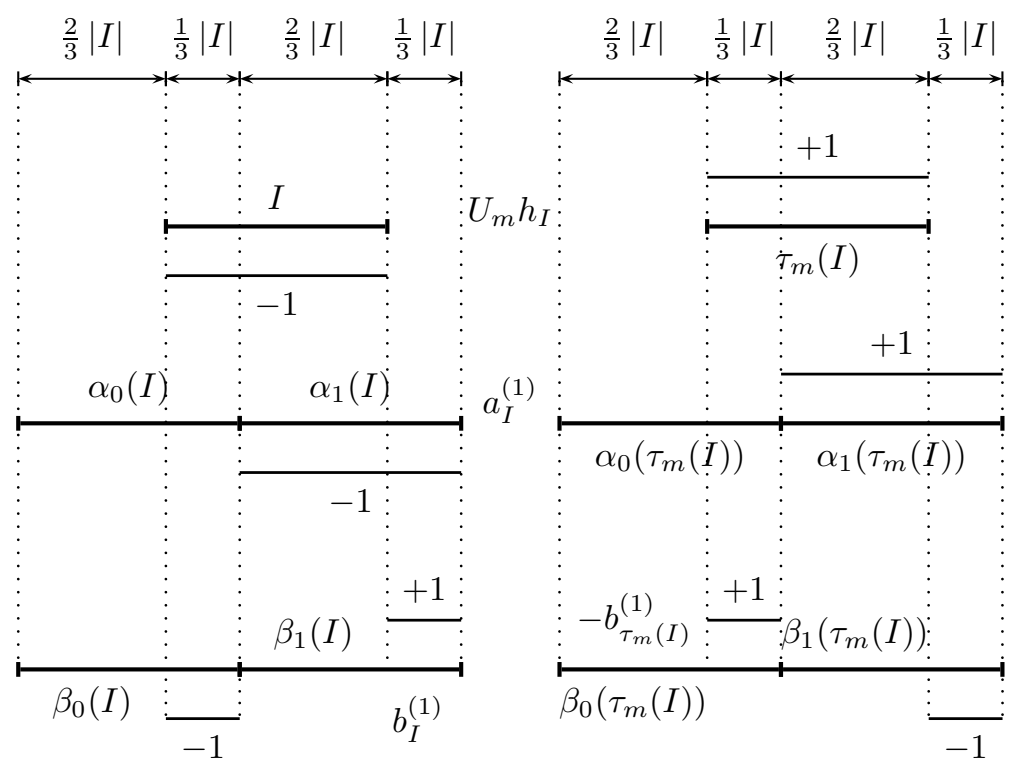

Figure 7. Martingale decomposition of $U_{m}$ to the right.

where

$$
\begin{aligned}
& \mathscr{B}_{i}^{(1,0)}=\left\{I \in \mathscr{B}_{i}^{(1)}: \inf \tau_{m}(I) \neq \inf \pi^{\lambda}\left(\tau_{m}(I)\right)\right\}, \\
& \mathscr{B}_{i}^{(1,1)}=\left\{I \in \mathscr{B}_{i}^{(1)}: \inf \tau_{m}(I)=\inf \pi^{\lambda}\left(\tau_{m}(I)\right)\right\},
\end{aligned}
$$

for all $0 \leq i \leq K(m)$. The projections

$$
P_{i}^{(0)} u=\sum_{I \in \mathscr{B}_{i}^{(0)}}\left\langle u, h_{I}\right\rangle h_{I}|I|^{-1}
$$

were defined in (4.16), accordingly we set

$$
P_{i}^{(1, \varepsilon)} u=\sum_{I \in \mathscr{B}_{i}^{(1, \varepsilon)}}\left\langle u, h_{I}\right\rangle h_{I}|I|^{-1},
$$

for all $0 \leq i \leq K(m)$ and $\varepsilon \in\{0,1\}$. The collection $\mathscr{B}_{i}^{(0)}$ is specified in Lemma 4.1. and $\mathscr{B}_{i}^{(1, \varepsilon)}$ is defined in (5.19) and (5.20). Finally, if we define

$$
\begin{aligned}
P^{(0)} & =\sum_{i=0}^{K(m)} P_{i}^{(0)}, \\
P^{(1, \varepsilon)} & =\sum_{i=0}^{K(m)} P_{i}^{(1, \varepsilon)},
\end{aligned}
$$

for all $\varepsilon \in\{0,1\}$, then certainly

$$
u=P^{(0)} u+P^{(1,0)} u+P^{(1,1)} u
$$

for all $u \in L_{X}^{p}$. Note that $P_{i}^{(1)}=P_{i}^{(1,0)}+P_{i}^{(1,1)}$, where $P_{i}^{(1)}$ was defined in (4.16).

In the following theorem the operator $U_{m}$ is decomposed into five parts, each of which is forming a martingale difference sequence. 
Theorem 5.1. Let $m \in \mathbb{Z}, m \geq 1$ and $0 \leq i \leq K(m)$. Then the identity

$$
U_{m} u=U_{m} \circ P^{(0)} u+\sum_{\varepsilon \in\{0,1\}}\left(A_{m}^{(\varepsilon)}+B_{m}^{(\varepsilon)}\right) \circ P^{(1, \varepsilon)} u
$$

holds true for all $u \in L_{X}^{p}$. For every $0 \leq i \leq K(m)$ and $\varepsilon \in\{0,1\}$, each of the following collections is a martingale difference sequence:

$$
\begin{aligned}
& \left\{U_{m} \circ P_{i}^{(0)} h_{I}: I \in \mathscr{D}\right\}, \\
& \left\{A_{m}^{(\varepsilon)} \circ P_{i}^{(1, \varepsilon)} h_{I}: I \in \mathscr{D}\right\} \text {, } \\
& \left\{B_{m}^{(\varepsilon)} \circ P_{i}^{(1, \varepsilon)} h_{I}: I \in \mathscr{D}\right\} .
\end{aligned}
$$

We have the estimate $K(m) \leq 7+2 \cdot \log _{2}(m)$, where $K(m)$ is defined in Lemma 4.1 on page 8 ,

Remark 5.2. For reasons of symmetry, a similar result holds true for $m \leq-1$, when adjusting the construction of $a_{I}, b_{I}$ and $\mathscr{B}_{i}^{(1, \varepsilon)}$, accordingly.

Proof. Let $m \in \mathbb{Z}, m \geq 1$ and $0 \leq i \leq K(m)$ be fixed throughout the rest of this proof. Whenever we apply the predecessor map $\pi$ to an interval $I \in \sigma^{\delta}(\mathscr{D})$, we understand it with respect to $\sigma^{\delta}(\mathscr{D})$, with $\delta \in\{0,1\}$ fixed.

Observe, identity (5.24) follows immediately from (5.23) and (5.17).

First, note that Lemma 4.1 implies that

$$
\left\{I, \tau_{m}(I), I \cup \tau_{m}(I): I \in \mathscr{B}_{i}^{(0)}\right\}
$$

is a nested collection of sets, hence

$$
\left\{U_{m} h_{I}: I \in \mathscr{B}_{i}^{(0)}\right\}
$$

is a martingale difference sequence.

Secondly, we will show that $\left\{a_{I}^{(0)}: I \in \mathscr{B}_{i}^{(1,0)}\right\}$ forms a martingale difference sequence. Henceforth, we shall abbreviate $\mathscr{B}_{i}^{(1,0)}$ by $\mathscr{B}$. Now, fix $I, J \in \mathscr{B},|J|<|I|$ such that $\operatorname{supp} a_{J}^{(0)} \cap \operatorname{supp} a_{I}^{(0)} \neq \emptyset$. Note that $J \subset\left(\pi^{\lambda}(J)\right)_{11}$, for all $J \in \mathscr{B}$, where $K_{11}, K \in \mathscr{D}$ denotes the unique $M \subset K, M \in \mathscr{D},|M|=|K| / 4$ such that $\sup M=$ $\sup K$. From this and the definition of $\mathscr{B}$ it is clear that $\operatorname{supp} a_{J}^{(0)} \subset \alpha_{1}\left(\pi^{\lambda}(J)\right)$ (see also Remark 4.2), hence

$$
\emptyset \neq \alpha_{1}\left(\pi^{\lambda}(J)\right) \cap \operatorname{supp} a_{I}^{(0)}=\left(\alpha_{1}\left(\pi^{\lambda}(J)\right) \cap \alpha_{0}(I)\right) \cup\left(\alpha_{1}\left(\pi^{\lambda}(J)\right) \cap \alpha_{0}\left(\tau_{m}(I)\right)\right) .
$$

Since $|J|<|I|, I, J \in \mathscr{B}$, we know that $\left|\alpha_{1}\left(\pi^{\lambda}(J)\right)\right| \leq|I|$, thus

$$
\text { either } \alpha_{1}\left(\pi^{\lambda}(J)\right) \subset \alpha_{0}(I) \text { or } \quad \alpha_{1}\left(\pi^{\lambda}(J)\right) \subset \alpha_{0}\left(\tau_{m}(I)\right) \text {, }
$$

which finishes the second part of this proof.

The proof that $\left\{a_{I}^{(1)}: I \in \mathscr{B}_{i}^{(1,1)}\right\}$ forms a martingale difference sequence is essentially the same, and we omit the details.

Thirdly, we will show that $\left\{b_{I}^{(0)}, b_{\tau_{m}(I)}^{(0)}: I \in \mathscr{B}^{(1,0)}\right\}$ constitutes a martingale difference sequence. Again, we shall abbreviate $\mathscr{B}_{i}^{(1,0)}$ by $\mathscr{B}$. To this end, we assume there exist $I, J \in \mathscr{B} \cup \tau_{m}(\mathscr{B}),|J|<|I|$ such that

$$
\beta(J) \cap \beta(I) \neq \emptyset \quad \text { and } \quad \beta(J) \cap \beta(I)^{c} \neq \emptyset .
$$

Since $\beta(J) \subset \gamma(J)$, assumption ( $(\mathcal{A})$ is covered by the following four cases.

(1) $\gamma(J) \cap I \neq \emptyset$ and $\gamma(J) \cap I^{c} \neq \emptyset$,

(2) $\gamma(J) \cap \gamma_{0}(I) \neq \emptyset$ and $\gamma(J) \cap \gamma_{0}(I)^{c} \neq \emptyset$,

(3) $\gamma(J) \subset I$ and inf $\beta_{1}(I) \in \gamma(J)$,

(4) $\gamma(J) \subset \gamma_{0}(I)$ and $\inf \beta_{0}(I) \in \gamma(J)$. 
If we assume case (1), then $\inf J=\inf I$ or $\inf J=\sup I$. Anyhow, we have that $\inf J=\inf \pi^{\lambda}(J)$, so we know $J \notin\left(\mathscr{B} \cup \tau_{m}(\mathscr{B})\right)$, contradicting our assumption. Case (2) is analogous to case (11). Note that we abbreviated $\mathscr{B}_{i}^{(1,0)}$ by $\mathscr{B}$, so consider the definition of $\mathscr{B}_{i}^{(1)}$ to see that $J \notin \mathscr{B}_{i}^{(1,0)}$, and consider (5.19) to determine that also $J \notin \tau_{m}\left(\mathscr{B}_{i}^{(1,0)}\right)$.

Let us now assume case (3) is true. This means that either inf $I+\frac{1}{3}|I| \in \gamma(J)$ or $\inf I+\frac{2}{3}|I| \in \gamma(J)$, depending on the sign of the one-third-shift for $I$. We fix $z \in\{1,2\}$ and assume that

$$
\inf I+\frac{z}{3}|I| \in \gamma(J) .
$$

Due to (5.19) we see that $\pi^{\lambda}\left(\gamma_{0}(J)\right)=\pi^{\lambda}(J)$, so if we set $K=\pi^{\lambda}(J)$, then

$$
\inf I+\frac{z}{3}|I| \in K \text {. }
$$

This corresponds to either one of the following being true

$$
\inf I+\frac{z}{3}|I|=\inf K+\frac{1}{3}|K| \quad \text { or } \quad \inf I+\frac{z}{3}|I|=\inf K+\frac{2}{3}|K| .
$$

If $J \in \mathscr{B}$ we know $J \subset K_{11}$, thus

$$
\begin{aligned}
\inf \gamma(J) & \geq \inf K+\frac{3}{4}|K|-2^{-\lambda}|K| \\
& >\inf K+\frac{2}{3}|K| .
\end{aligned}
$$

Recall that $K_{11}$ denotes the unique $M \subset K, M \in \mathscr{D},|M|=|K| / 4$ such that $\sup M=\sup K$. The last strict inequality holds true since $\lambda \geq 4$ per construction of $\mathscr{B}$, see (4.7) if $|m| \geq 2$ and note the exception for $|m|=1$ beneath. Combining (5.28) and (5.30) yields

$$
\inf I+\frac{z}{3}|I|>\inf K+\frac{2}{3}|K|
$$

which contradicts (5.29) in both cases.

If $J \in \tau_{m}(\mathscr{B})$ we know $J \subset K_{00}$, where $K_{00}$ denotes the unique $M \subset K, M \in \mathscr{D}$, $|M|=|K| / 4$ such that $\inf M=\inf K$. So we note

$$
\begin{aligned}
\sup \gamma(J) & \leq \inf K+\frac{1}{4}|K|+2^{-\lambda}|K| \\
& <\inf K+\frac{1}{3}|K| .
\end{aligned}
$$

The last strict inequality holds true since $\lambda \geq 4$ per construction of $\mathscr{B}$, see (4.7) if $|m| \geq 2$ and note the exception for $|m|=1$ beneath. Combining (5.28) and (5.31) yields

$$
\inf I+\frac{z}{3}|I|<\inf K+\frac{1}{3}|K|
$$

which contradicts (5.29) in both cases.

Case (4) is analogous to case (3).

Altogether we proved that our assumption $(\mathcal{A})$ was false, therefore

$$
\beta(J) \subset \beta_{0}(I) \quad \text { or } \quad \beta(J) \subset \beta_{1}(I)
$$

for all $I, J \in \mathscr{B},|J|<|I|$ such that $\beta(J) \cap \beta(I) \neq \emptyset$. In other words, the support of $b_{J}$ is contained in a set where $b_{I}^{(0)}$ is constant, hence

$$
\left\{b_{I}^{(0)}, b_{\tau_{m}(I)}^{(0)}: I \in \mathscr{B}_{i}^{(1,0)}\right\}
$$

constitutes a martingale difference sequence. 
The proof that $\left\{b_{I}^{(1)}, b_{\tau_{m}(I)}^{(1)}: I \in \mathscr{B}^{(1,1)}\right\}$ constitutes a martingale difference sequence is essentially the same argument, so we omit it.

Remark 5.3. Note in Theorem5.1 we actually proved the following stronger result. For every $0 \leq i \leq K(m)$ and $\varepsilon \in\{0,1\}$, the collection

$$
\left\{b_{I}^{(\varepsilon)}, b_{\tau_{m}(I)}^{(\varepsilon)}: I \in \mathscr{B}_{i}^{(1, \varepsilon)}\right\}
$$

is a martingale difference sequence, which certainly implies (5.27).

Consider the splitting of $\mathscr{D}$ into the sets $\mathscr{B}_{i}^{(\delta)}, 0 \leq i \leq K(m), \delta \in\{0,1\}$, see Lemma 4.1 on page 8 for details, which we used in Theorem 4.3 on page 10 to treat the shift operator $T_{m}$. Retracing our steps in the proof of Theorem 4.3 we find that we could actually repeat this proof with the operator $T_{m}$ replaced by any of the operators $U_{m} \circ P^{(0)}, A_{m}^{(\varepsilon)} \circ P^{(1, \varepsilon)}, B_{m}^{(\varepsilon)} \circ P^{(1, \varepsilon)}, \varepsilon \in\{0,1\}$. The details are elaborated in Theorem 5.4 below.

Theorem 5.4. Let $m \in \mathbb{Z}$ and $m \geq 1$. Then for all $0 \leq i \leq K(m)$ and $\varepsilon \in\{0,1\}$, we have the estimates

$$
\begin{gathered}
\left\|U_{m} \circ P_{i}^{(0)} u\right\|_{L_{X}^{p}} \leq C \cdot\left\|P_{i}^{(0)} u\right\|_{L_{X}^{p}}, \\
\left\|U_{m} \circ P_{i}^{(1, \varepsilon)} u\right\|_{L_{X}^{p}} \leq C \cdot\left\|P_{i}^{(1, \varepsilon)} u\right\|_{L_{X}^{p}},
\end{gathered}
$$

for all $u \in L_{X}^{p}$, where the constant $C$ depends only on $\mathscr{U}_{p}(X)$. Furthermore, we have the bound $K(m) \leq 7+2 \cdot \log _{2}(m)$.

Remark 5.5. For reasons of symmetry, the same result holds true for $m \leq-1$, that is besides the appropriate modifications for $P_{i}^{(0)}$ and $P_{i}^{(1, \varepsilon)}$.

Proof. Let $m \in \mathbb{Z}, m \geq 1$ and $0 \leq i \leq K(m)$ be fixed throughout the rest of the proof.

First, we will estimate $U_{m} \circ P_{i}^{(0)}$. Due to Theorem 5.1 respectively Remark 5.3 we know that $\left\{U_{m} \circ P_{i}^{(0)} h_{I}: I \in \mathscr{D}\right\}$ forms a martingale difference sequence, which enables us to introduce Rademacher functions via the UMD-property. Hence

$$
\begin{aligned}
\left\|U_{m} \circ P_{i}^{(0)} u\right\|_{L_{X}^{p}} & \approx \int_{0}^{1}\left\|\sum_{I \in \mathscr{B}_{i}^{(0)}} r_{I}(t)\left\langle u, h_{I}\right\rangle U_{m} h_{I}|I|^{-1}\right\|_{L_{X}^{p}} \mathrm{~d} t \\
& =\int_{0}^{1}\left\|\sum_{I \in \mathscr{B}_{i}^{(0)}} r_{I}(t)\left\langle u, h_{I}\right\rangle\left(\mathrm{Id}+T_{m}\right) h_{I}|I|^{-1}\right\|_{L_{X}^{p}} \mathrm{~d} t
\end{aligned}
$$

for all $u \in L_{X}^{p}$. This is all we need to repeat the proof of Theorem 4.3 in Section 4 with $T_{m}$ replaced by $\mathrm{Id}+T_{m}$.

Now we turn to the estimate for $U_{m} \circ P_{i}^{(1, \varepsilon)}$, with $\varepsilon \in\{0,1\}$ fixed throughout the rest of the proof. Observe that

$$
U_{m} \circ P_{i}^{(1, \varepsilon)} u=A_{m}^{(\varepsilon)} \circ P_{i}^{(1, \varepsilon)} u+B_{m}^{(\varepsilon)} \circ P_{i}^{(1, \varepsilon)} u,
$$

for all $u \in L_{X}^{p}$, see (5.17). Theorem 5.1 on page 16 ensures that both

$$
\left\{A_{m}^{(\varepsilon)} \circ P_{i}^{(1, \varepsilon)} h_{I}: I \in \mathscr{D}\right\} \quad \text { and } \quad\left\{B_{m}^{(\varepsilon)} \circ P_{i}^{(1, \varepsilon)} h_{I}: I \in \mathscr{D}\right\}
$$

form martingale difference sequences, which allows us to introduce Rademacher means via the UMD-property, hence

$$
\left\|A_{m}^{(\varepsilon)} \circ P_{i}^{(1, \varepsilon)} u\right\|_{L_{X}^{p}} \lesssim \int_{0}^{1}\left\|\sum_{I \in \mathscr{B}_{i}^{(1, \varepsilon)}} r_{I}(t)\left\langle u, h_{I}\right\rangle a_{I}^{(\varepsilon)}|I|^{-1}\right\|_{L_{X}^{p}} \mathrm{~d} t
$$


and

$$
\left\|B_{m}^{(\varepsilon)} \circ P_{i}^{(1, \varepsilon)} u\right\|_{L_{X}^{p}} \lesssim \int_{0}^{1}\left\|\sum_{I \in \mathscr{B}_{i}^{(1, \varepsilon)}} r_{I}(t)\left\langle u, h_{I}\right\rangle\left(b_{I}^{(\varepsilon)}-b_{\tau_{m}(I)}^{(\varepsilon)}\right) h_{I}|I|^{-1}\right\|_{L_{X}^{p}} \mathrm{~d} t
$$

for all $u \in L_{X}^{p}$. Now we can essentially repeat the proof of Theorem 4.3 in Section 4 , for $\delta=1$ and with $T_{m}$ replaced by $A_{m}^{(\varepsilon)}$ and $B_{m}^{(\varepsilon)}$, respectively. We have to utilize the unilateral operators $S_{0}$ and $S_{1}$ instead of $S$ as well, see Subsection 3.2 on page 6 . If we do so, we end up with the estimates

$$
\left\|A_{m}^{(\varepsilon)} \circ P_{i}^{(1, \varepsilon)} u\right\|_{L_{X}^{p}} \lesssim \int_{0}^{1}\left\|\sum_{I \in \mathscr{B}_{i}^{(1, \varepsilon)}} r_{I}(t)\left\langle u, h_{I}\right\rangle h_{\alpha_{\varepsilon}(I)}|I|^{-1}\right\|_{L_{X}^{p}} \mathrm{~d} t
$$

and

$$
\left\|B_{m}^{(\varepsilon)} \circ P_{i}^{(1, \varepsilon)} u\right\|_{L_{X}^{p}} \lesssim \int_{0}^{1}\left\|\sum_{I \in \mathscr{B}_{i}^{(1, \varepsilon)}} r_{I}(t)\left\langle u, h_{I}\right\rangle b_{I}^{(\varepsilon)}|I|^{-1}\right\|_{L_{X}^{p}} \mathrm{~d} t
$$

for all $u \in L_{X}^{p}$. Thus, considering $h_{\alpha_{\varepsilon}(I)}=S_{\varepsilon} h_{I}$ and $\left|b_{I}^{(\varepsilon)}\right| \leq\left|S_{0} h_{I}\right|+\left|S_{1} h_{I}\right|$, see (3.9), (3.10), (3.11), (3.12) and combining our estimates for $A_{m}^{(\varepsilon)}$ and $B_{m}^{(\varepsilon)}$ with the inequalities for the unilateral one-third-shift operators $S_{0}$ and $S_{1}$ in Theorem 3.3 on page 7 yields

$$
\begin{aligned}
\left\|U_{m} \circ P_{i}^{(1, \varepsilon)} u\right\|_{L_{X}^{p}} & \lesssim\left\|S_{0} \circ P_{i}^{(1, \varepsilon)} u\right\|_{L_{X}^{p}}+\left\|S_{1} \circ P_{i}^{(1, \varepsilon)} u\right\|_{L_{X}^{p}} \\
& \lesssim\left\|P_{i}^{(1, \varepsilon)} u\right\|_{L_{X}^{p}}
\end{aligned}
$$

for all $u \in L_{X}^{p}$, concluding the proof.

From the results established in Theorem 4.3 on page 10 and Theorem 5.4 on the facing page one can obtain the estimates stated in Theorem 5.6 below, by exploiting the type and cotype inequalities for $T_{m}$, and only the cotype inequality for $U_{m}$. Inserting Theorem 4.3 on page 10 and Theorem 5.4 on the facing page into Fig88, Lemma 1] one can obtain [Fig88, Theorem 1] stated below for sake of completeness.

Theorem 5.6 (Fig88). Let $1<p<\infty$, and $X$ be a Banach space with the $\mathrm{UMD}$-property. For $m \in \mathbb{Z}$ let the map $\tau_{m}$ denote the shift map defined by

$$
I \mapsto I+m|I|
$$

Let $T_{m}, U_{m}$ denote the linear extensions of the maps

$$
T_{m} h_{I}=h_{\tau_{m}(I)}
$$

and

$$
U_{m} h_{I}=1_{\tau_{m}(I)}-1_{I},
$$

respectively, then

$$
\begin{gathered}
\left\|T_{m}: L_{X}^{p} \rightarrow L_{X}^{p}\right\| \leq C\left(\log _{2}(2+|m|)\right)^{\alpha}, \\
\left\|U_{m}: L_{X}^{p} \rightarrow L_{X}^{p}\right\| \leq C\left(\log _{2}(2+|m|)\right)^{\beta},
\end{gathered}
$$

where the constant $C>0$ depends only $\mathscr{U}_{p}(X)$ and $0<\alpha, \beta<1$. Moreover, if $L_{X}^{p}$ has type $\mathcal{T}$ and cotype $\mathcal{C}$, then one can take $\alpha=\frac{1}{\mathcal{T}}-\frac{1}{\mathfrak{C}}$ and $\beta=1-\frac{1}{\mathfrak{e}}$. 


\section{REFERENCES}

[Bou86] J. Bourgain. Vector-valued singular integrals and the $H^{1}$-BMO duality. Probability theory and harmonic analysis, Pap. Mini-Conf., Cleveland/Ohio 1983, Pure Appl. Math., Marcel Dekker 98, 1-19 (1986)., 1986.

[Bur81] D. L. Burkholder. A Geometrical Characterization of Banach Spaces in which Martingale Difference Sequences are Unconditional. Annals of Probability, 9(6):997-1011, 1981.

[CWW85] S.Y.A. Chang, J.M. Wilson, and T.H. Wolff. Some weighted norm inequalities concerning the Schrödinger operators. Comment. Math. Helv., 60:217-246, 1985.

[Dav80] Burgess Davis. Hardy spaces and rearrangements. Trans. Amer. Math. Soc., 261(1):211-233, 1980.

[Fig88] T. Figiel. On Equivalence of Some Bases to the Haar System in Spaces of Vector-valued Functions. Bulletin of the Polish Academy of Sciences, 36(3-4):119-131, 1988.

[Fig90] T. Figiel. Singular Integral Operators: A Martingale Approach. In Geometry of Banach Spaces, number 158 in London Mathematical Society Lecture Note Series, pages 95110, 1990.

[FW01] T. Figiel and P. Wojtaszczyk. Special bases in function spaces. In Handbook of the geometry of Banach spaces, Vol. I, pages 561-597. North-Holland, Amsterdam, 2001.

[GJ82] John B. Garnett and Peter W. Jones. BMO from dyadic BMO. Pac. J. Math., 99:351371,1982

[Hyt11] T. P. Hytonen. Foundations of vector-valued singular integrals revisited-with random dyadic cubes. http://arxiv.org/abs/1110.5826, [v1] Wed, 26 Oct 2011, 2011.

[Kah85] J.-P. Kahane. Some Random Series of Functions, Second Edition. Cambridge University Press, 1985.

[Lec11] R. Lechner. An Interpolatory Estimate and Shift Operators. Ph.D. thesis, July 2011, http://shrimp.bayou.uni-linz.ac.at/Papers/dvi/phd_thesis_Richard_Lechner.pdf, 2011.

[MP11] Paul F. X. Müller and Markus Passenbrunner. A decomposition theorem for singular integral operators on spaces of homogeneous type. To appear in the Journal of Functional Analysis, 2011.

[Mül05] Paul F. X. Müller. Isomorphisms between $H^{1}$ spaces. Monografie Matematyczne. Instytut Matematyczny PAN (New Series) 66. Basel: Birkhäuser., 2005.

[NS97] I. Novikov and E. Semenov. Haar series and linear operators. Kluwer Academic Publishers, 1997.

[NTV97] F. Nazarov, S. Treil, and A. Volberg. Cauchy integral and Calderón-Zygmund operators on nonhomogeneous spaces. Internat. Math. Res. Notices, 15:703-726, 1997.

[NTV03] F. Nazarov, S. Treil, and A. Volberg. The $T b$-theorem on non-homogeneous spaces. Acta Math., 190(2):151-239, 2003.

[Ste70] E. M. Stein. Topics in harmonic analysis related to the Littlewood-Paley theory. Annals of Mathematics Studies, No. 63. Princeton University Press, Princeton, N.J., 1970.

[Wol82] Thomas H. Wolff. Two algebras of bounded functions. Duke Math. J., 49:321-328, 1982 .

Institute of AnAlysis

Johannes Kepler University Linz

Altenbergerstrasse 69

A-4040 Linz, Austria 